ESAIM: M2AN 49 (2015) 815-837

DOI: $10.1051 / \mathrm{m} 2 \mathrm{an} / 2014050$
ESAIM: Mathematical Modelling and Numerical Analysis

www.esaim-m2an.org

\title{
DISCRETE LEAST SQUARES POLYNOMIAL APPROXIMATION WITH RANDOM EVALUATIONS - APPLICATION TO PARAMETRIC AND STOCHASTIC ELLIPTIC PDES
}

\author{
Abdellah Chkifa ${ }^{1}$, Albert Cohen $^{1}$, Giovanni Migliorati ${ }^{2}$, \\ Fabio Nobile ${ }^{2}$ and Raul Tempone ${ }^{3}$
}

\begin{abstract}
Motivated by the numerical treatment of parametric and stochastic PDEs, we analyze the least-squares method for polynomial approximation of multivariate functions based on random sampling according to a given probability measure. Recent work has shown that in the univariate case, the leastsquares method is quasi-optimal in expectation in [A. Cohen, M A. Davenport and D. Leviatan. Found. Comput. Math. 13 (2013) 819-834] and in probability in [G. Migliorati, F. Nobile, E. von Schwerin, R. Tempone, Found. Comput. Math. 14 (2014) 419-456], under suitable conditions that relate the number of samples with respect to the dimension of the polynomial space. Here "quasi-optimal" means that the accuracy of the least-squares approximation is comparable with that of the best approximation in the given polynomial space. In this paper, we discuss the quasi-optimality of the polynomial least-squares method in arbitrary dimension. Our analysis applies to any arbitrary multivariate polynomial space (including tensor product, total degree or hyperbolic crosses), under the minimal requirement that its associated index set is downward closed. The optimality criterion only involves the relation between the number of samples and the dimension of the polynomial space, independently of the anisotropic shape and of the number of variables. We extend our results to the approximation of Hilbert space-valued functions in order to apply them to the approximation of parametric and stochastic elliptic PDEs. As a particular case, we discuss "inclusion type" elliptic PDE models, and derive an exponential convergence estimate for the least-squares method. Numerical results confirm our estimate, yet pointing out a gap between the condition necessary to achieve optimality in the theory, and the condition that in practice yields the optimal convergence rate.
\end{abstract}

Mathematics Subject Classification. 41A10, 41A25, 65N35, 65N12, 65N15, 35J25.

Received November 5, 2013. Revised July 20, 2014.

Published online 8 April 2015.

\footnotetext{
Keywords and phrases. Approximation theory, polynomial approximation, least squares, parametric and stochastic PDEs, highdimensional approximation.

1 Institut Universitaire de France, UPMC Univ Paris 06, UMR 7598, Laboratoire Jacques-Louis Lions, 75005 Paris, France. chkifa@ann.jussieu.fr; cohen@ann.jussieu.fr

2 MATHICSE-CSQI, École Polytechnique Fédérale de Lausanne, 1015 Lausanne, Switzerland giovanni.migliorati@epfl.ch; fabio.nobile@epfl.ch

3 Applied Mathematics and Computational Sciences, and SRI Center for Uncertainty Quantification in Computational Science and Engineering, KAUST, 23955-6900 Thuwal, Saudi Arabia. raul.tempone@kaust.edu.sa
} 


\section{INTRODUCTION}

In recent years, various strategies have been proposed for the numerical treatment of parametric and stochastic partial differential equations

$$
\mathcal{D}(u, y)=0,
$$

where $u \mapsto \mathcal{D}(u, y)$ is a partial differential operator depending on a $d$-dimensional parameter vector

$$
y:=\left(y_{1}, \ldots, y_{d}\right) \in \Gamma \subset \mathbb{R}^{d} .
$$

Depending on the application, the parameter vector may be deterministic or stochastic. In the latter case $y$ is a random variable distributed over $\Gamma$ according to a probability measure $\rho$. We denote by $(\Gamma, \Sigma, \rho)$ the corresponding probability space, where $\Sigma$ is the Borel $\sigma$-algebra. In certain applications one has to deal with a countable number of parameters $y=\left(y_{j}\right)_{j \geq 1}$ which means that $d=+\infty$.

Assuming well-posedness of the problem in some Banach space $X$, the solution map

$$
y \mapsto u(y),
$$

is defined from the parameter domain $\Gamma$ to the solution space $X$. In both deterministic and stochastic settings, the main challenge is to approximate the function $y \mapsto u(y)$ with a reasonable cost. In the first setting, one typically searches for approximations that are uniformly accurate over the parameter space $\Gamma$, which amounts in measuring the error in $L^{\infty}(\Gamma, X)$. In the second setting, one is typically interested in approximations that are accurate in a probabilistic sense, such as in the least-squares sense which amounts in measuring the error in $L^{2}(\Gamma, X, \rho)$.

Polynomial approximation methods of the solution map have been studied for various types of operators $\mathcal{D}$ corresponding to various PDEs. In such methods, the solution map is approximated by polynomial maps of the form

$$
u_{\Lambda}(y)=\sum_{\nu \in \Lambda} u_{\nu} y^{\nu}
$$

where $\Lambda \subset \mathcal{F}$ is a finite set of (multi-)indices. The set of multi-indices $\mathcal{F}$ coincides with $\mathbb{N}_{0}^{d}$ where $\mathbb{N}_{0}=\{0,1,2, \ldots\}$ in the case $d<+\infty$ and denote the countable set of all finitely supported sequences $\nu=\left(\nu_{1}, \nu_{2}, \ldots, 0,0, \ldots\right) \in \mathbb{N}_{0}^{\mathbb{N}}$ in the case $d=+\infty$. Also, in both cases, the polynomials $y \mapsto y^{\nu}$ are defined by

$$
y^{\nu}:=\prod_{j=1}^{d} y_{j}^{\nu_{j}},
$$

with the convention $0^{0}=1$. Note that the coefficients $u_{\nu}$ belong to the Banach space $X$ and therefore the construction of $u_{\Lambda}$ requires in principle the computation of $\#(\Lambda)$ such functions. The functions $u_{\Lambda}$ are thus selected in $X_{\Lambda}:=X \otimes \mathbb{P}_{\Lambda}$, where

$$
\mathbb{P}_{\Lambda}:=\operatorname{Span}\left\{y^{\nu}: \nu \in \Lambda\right\}
$$

denotes the polynomial space associated with the index set $\Lambda$ and with coefficients in $\mathbb{R}$. Throughout this paper, we only work with index sets $\Lambda$ that have the following natural property.

Definition 1.1. The index set $\Lambda \subset \mathcal{F}$ is downward closed if

$$
\nu \in \Lambda \quad \text { and } \quad \nu^{\prime} \leq \nu \Rightarrow \nu^{\prime} \in \Lambda,
$$

where $\nu^{\prime} \leq \nu$ means that $\nu_{j}^{\prime} \leq \nu_{j}$ for all $j \geq 1$. 
Following a more concise and established terminology in the literature, we will also denote by lower set a downward closed set. Note that a lower set always contains the null index

$$
0_{\mathcal{F}}:=(0,0, \ldots)
$$

Considering only polynomial spaces $\mathbb{P}_{\Lambda}$ associated with such sets is very natural. In particular, the downward closedness property of the set $\Lambda$ allows us to replace the monomials $y^{\nu}$ in the definition of the spaces $\mathbb{P}_{\Lambda}$ by any other tensorized basis of the form $P_{\nu}(y)=\prod_{j \geq 1} P_{\nu_{j}}\left(y_{j}\right)$ where $\left(P_{k}\right)_{k \geq 0}$ is a sequence of univariate polynomials such that $P_{0}=1$ and $P_{k}$ has degree exactly equal to $k$, for example the Legendre polynomials. Polynomial spaces associated with lower index sets have been introduced in [16] in dimension $d=2$ and in [17] and [13] in higher dimension.

Polynomial approximation is well known to be effective when the solution map has some smoothness. In certain instances, it can even provably break the curse of dimensionality, in the sense that an algebraic convergence rate with respect to $\#(\Lambda)$ can be established even for functions of countably many parameters $d=+\infty$. Such results are proven in $[5,9,10]$ for the model parametric elliptic equation

$$
-\operatorname{div}(a \nabla u)=f \text { in } D \subset \mathbb{R}^{q}, u=0 \text { on } \partial D,
$$

where $D \subset \mathbb{R}^{q}$ is a Lipschitz domain, $f \in H^{-1}(D)$, and the diffusion coefficient has the form

$$
a(x, y):=\bar{a}(x)+\sum_{j \geq 1} y_{j} \psi_{j}(x)
$$

with the functions $\psi_{j}$ and $\bar{a}$ in $L^{\infty}(D)$, and $y \in \Gamma:=[-1,1]^{\mathbb{N}}$. Assuming the uniform ellipticity assumption

$$
0<r \leq a(x, y) \leq R<+\infty, \quad x \in D, y \in \Gamma,
$$

the solution map is well defined from $\Gamma$ to the Hilbert space $X:=H_{0}^{1}(D)$. Then, it is proved in [5] that if $\left(\left\|\psi_{j}\right\|_{L^{\infty}}\right)_{j \geq 1} \in \ell^{p}(\mathbb{N})$ for some $0<p<1$, there exists a sequence of lower sets

$$
\Lambda_{1} \subset \Lambda_{2} \subset \ldots \subset \mathcal{F}, \quad \#\left(\Lambda_{m}\right)=m
$$

such that

$$
\inf _{v \in X_{\Lambda_{m}}}\|u-v\|_{L^{\infty}(\Gamma, X)} \leq C m^{-s}, \quad s:=\frac{1}{p}-1>0 .
$$

Similar results with a slightly improved convergence rate are obtained in $[7,9,10]$ for the $L^{2}(\Gamma, X, \rho)$ norm, where $\rho$ denotes the uniform probability measure: under the same assumptions there exists a sequence of lower sets such that

$$
\inf _{v \in X_{\Lambda_{m}}}\|u-v\|_{L^{2}(\Gamma, X, \rho)} \leq C m^{-s}, \quad s:=\frac{1}{p}-\frac{1}{2}>0 .
$$

These general convergence results are extended in [6] to other models than (1.9).

The construction of sequences of sets $\left(\Lambda_{m}\right)_{m \geq 1}$ which achieve the convergence rates (1.13) or (1.14), and therefore of the polynomial spaces $\mathbb{P}_{\Lambda_{m}}$, is critical in the design of algorithms for high-dimensional approximation. Sequences of quasi-optimal sets giving such rates, with possibly a suboptimal constant $C>0$ can either be derived from a priori estimates in $[3,6,7,9,10]$ or by an adaptive search $[5,6,14]$. The resulting spaces $\mathbb{P}_{\Lambda_{m}}$ typically differ from the standard multivariate polynomial spaces $\mathbb{P}_{k}$ of fixed total degree. 
Given a finite index set $\Lambda$, several strategies can be used to compute $u_{\Lambda} \in X_{\Lambda}$ :

1. Taylor expansions [5] can be recursively computed in the case of problems with affine parameter dependence such as (1.9). Adaptive methods based on such expansions have been proved to converge uniformly with the same rate as in (1.13).

2. Projection methods $[2,3,9,14]$ produce quasi-optimal approximations in $X_{\Lambda}$ for the metric $L^{2}(\Gamma, X, \rho)$ where $\rho$ is a chosen measure in the parameter space. In addition, in the Galerkin framework, it is possible to use techniques of a posteriori analysis in order to adaptively build the sequence of index sets $\left(\Lambda_{m}\right)_{m \geq 1}$. This approach was developed in [14] for the problem (1.9), and proved to converge with the same rate as in (1.14).

3. Collocation methods $[1,3,6,20,23,24]$ produce a polynomial approximation in $X_{\Lambda}$ based on the data of particular solution instances $u^{i}:=u\left(y^{i}\right)$ for some chosen values $y^{i} \in \Gamma$ of the parameter vector with $i=1, \ldots, n$. One significant advantage of this approach is that it is non intrusive: the $u^{i}$ can be computed by any given numerical solver for the problem (1.1) and the polynomial approximation is built from these solutions by numerical techniques similar to those employed for scalar-valued maps such as interpolation or least-squares regression.

The convergence analysis of collocation methods is less satisfactory in the sense that convergence rates similar to (1.13) and (1.14) do not seem to have been established for such methods. This is in part due to the difficulty to control the stability of interpolation or least-squares projection for general multivariate polynomial spaces. For interpolation methods, several results have been recently established in [6] showing that the convergence rate in (1.13) can be achieved if the interpolation points are carefully selected. Least-squares methods have been recently analyzed in $[8,20]$ in the stochastic setting, assuming that the samples $y^{i}$ are independent realizations of the random variable $y$, therefore identically distributed according to $\rho$. This analysis reveals that in the univariate case $\Gamma=[-1,1]$ and for the uniform distribution, the least-squares method is stable with high probability under the condition that the number of samples $n$ scales quadratically (up to a logarithmic factor) with respect to the dimension $m$ of the polynomial space $\mathbb{P}_{m-1}$. By "stable", one means that the $L^{2}(\Gamma, \rho)$ of the least squares projection is bounded up to a fixed multiplicative constant by the $\ell^{2}$ norm of the discrete observations. This analysis also shows that the least squares method produces quasi-optimal approximations in the $L^{2}(\Gamma, \rho)$ norm, either with high probability or in expectation.

The objective of this paper is to address the problem of the stability and convergence of the multivariate polynomial least-squares method in the general context of the spaces $X_{\Lambda}$ associated with arbitrary lower sets. The extension of the stability results given in $[8,20]$ to the multivariate case is not straightforward. One of our main results shows that the polynomial least-squares method with $\Gamma=[-1,1]^{d}$ is stable for any lower set $\Lambda$ and arbitrary dimension $d$, in the case of the uniform measure, under the same condition as in the univariate case. Namely, assuming that $n$ scales quadratically (up to a logarithmic factor) with respect to the dimension $\#(\Lambda)$ of the polynomial space, the least-square method is stable with probability at least $1-2 n^{-r}$ where $r>0$ can be taken arbitrarily large. We have also extended this result to more general measures from the beta family. The strength of this result is that the stability condition depends only on the cardinality of the set (provided it is downward closed) and not on its "shape". This allows us to establish effective quasi-optimal approximation results, even in infinite dimension, using suitable sequences of anisotropic lower sets.

The outline of the paper is as follows. We begin in Section 2 by discussing the least-squares method for realvalued functions in a general framework not limited to polynomials, recalling recent stability and approximation results established in [8], and introducing some variants for the case of noisy data. In Section 3 we focus on the particular framework of the multivariate polynomial spaces $\mathbb{P}_{\Lambda}$ and derive our stability and convergence results with $\Gamma=[-1,1]^{d}$ for any lower set $\Lambda$ and arbitrary dimension $d$. Then in Section 4, we show how a similar analysis applies to $X$-valued functions, where $X$ is a Hilbert space, and therefore to the exact or discretized solutions of parametric and stochastic PDEs. As a relevant example, the equation (1.9) with random inclusions in the diffusion coefficient is discussed in Section 5, and numerical illustration for this example are given in Section 6 . 


\section{Discrete least-SquARes approximations}

Let $(\Gamma, \Sigma, \rho)$ be a probability space. We denote by $L^{2}(\Gamma, \rho)$ the Hilbert space of real-valued square integrable functions with respect to $\rho$ and denote by $\langle\cdot, \cdot\rangle$ and $\|\cdot\|$ the associated inner product and norm, i.e.

$$
\langle v, w\rangle:=\int_{\Gamma} v(y) w(y) d \rho(y), \quad\|v\|:=\sqrt{\langle v, v\rangle}, \quad v, w \in L^{2}(\Gamma, \rho) .
$$

We consider $V_{m}$ a finite dimensional subspace of $L^{2}(\Gamma, \rho)$ with $\operatorname{dim}\left(V_{m}\right)=m$. We assume that the functions belonging to $V_{m}$ are defined everywhere over $\Gamma$. We let $\mathcal{B}_{L}:=\left(L_{j}\right)_{1 \leq j \leq m}$ be any orthonormal basis of $V_{m}$ with respect to the above inner product. The best approximation of a function $u \in L^{2}(\Gamma, \rho)$ in the least-squares sense is given by

$$
P_{m} u=\sum_{j=1}^{m} c_{j} L_{j}, \quad c_{j}=\left\langle u, L_{j}\right\rangle
$$

and its best approximation error by

$$
e_{m}(u):=\inf _{v \in V_{m}}\|u-v\|=\left\|u-P_{m} u\right\| .
$$

If $u$ is unknown and if $\left(z^{i}\right)_{i=1, \ldots, n}$ are noiseless or noisy observations of $u$ at the points $\left(y^{i}\right)_{i=1, \ldots, n}$ where the $y^{i}$ are i.i.d. random variables distributed according to $\rho$, we introduce the discrete least-squares approximation

$$
w:=\underset{v \in V_{m}}{\operatorname{argmin}} \sum_{i=1}^{n}\left|z^{i}-v\left(y^{i}\right)\right|^{2} .
$$

More precisely, the observation model is

$$
z^{i}=u\left(y^{i}\right)+\eta^{i}, \quad i=1, \ldots, m,
$$

where $y^{i}$ are i.i.d. random variable distributed according to $\rho$ and where $\eta^{i}$ represents the noise. Several scenarii may be considered for modeling the noise:

1. Noiseless model: one has $\eta^{i}=0$.

2. Stochastic noise model: $\eta^{i}$ are centered i.i.d. random variables, with uniformly bounded variance

$$
\sup _{y \in \Gamma} \mathbb{E}\left(|\eta|^{2} \mid y\right)<\infty .
$$

3. Deterministic noise model: $\eta^{i}=\eta\left(y_{i}\right)$ where $\eta$ is a uniformly bounded function on $\Gamma$ with

$$
\|\eta\|_{L^{\infty}(\Gamma)}<\infty
$$

In the framework of parametric PDE's, the observation noise represents the discretization error between the exact solution $u(y)$ and the solution computed by deterministic numerical solver, which is a function of $y$. The deterministic noise model is therefore the appropriate one, with $\|\eta\|_{L^{\infty}(\Gamma)}$ representing a uniform bound on the discretization error guaranteed by the numerical solver.

This minimization problem always has a solution, which may not be unique. In particular, it is never unique in the regime $m>n$. In the following, we only consider the regime $m \leq n$. In the noiseless case, $z^{i}=u\left(y^{i}\right)$, the solution may be viewed as the orthogonal projection of $u$ onto $V_{m}$ with respect to the inner product $\langle\cdot, \cdot\rangle_{n}$ associated with the empirical semi-norm

$$
\|v\|_{n}=\left(\frac{1}{n} \sum_{i=1}^{n}\left|v\left(y^{i}\right)\right|^{2}\right)^{\frac{1}{2}} .
$$


In this case, we denote the solution $w$ of the problem (2.4) by $P_{m}^{n} u$. The projection $P_{m}^{n} u$ depends on the sample $\left(y^{j}\right)_{1 \leq j \leq n}$, so that $P_{m}^{n}$ is a "random" least-squares projector. In both the noisy and noiseless case, the coordinate vector $\mathbf{w} \in \mathbb{R}^{m}$ of $w$ in the basis $\mathcal{B}_{L}$ is the solution to the system

$$
\mathbf{G w}=\mathbf{J z}
$$

where $\mathbf{G}$ and $\mathbf{J}$ are the $m \times m$ and $m \times n$ matrices given by

$$
\mathbf{G}_{i j}:=\left\langle L_{i}, L_{j}\right\rangle_{n}, \quad \text { and } \quad \mathbf{J}_{i j}:=\frac{L_{i}\left(y^{j}\right)}{n}
$$

and $\mathbf{z} \in \mathbb{R}^{n}$ is the vector of coordinates $z^{j}$. Note that

$$
n \mathbf{J J}^{t}=\mathbf{G} .
$$

When $\mathbf{G}$ is not singular, then the solution $w$ of (2.4) is given by

$$
w=\sum_{j=1}^{n} z^{j} \pi_{j} .
$$

where $\mathcal{B}_{\pi}:=\left\{\pi_{1}, \ldots, \pi_{n}\right\}$ are the elements of $V_{m}$ given by

$$
\mathcal{B}_{\pi}=\left(\mathbf{G}^{-1} \mathbf{J}\right)^{t} \mathcal{B}_{L}
$$

with the product matrix-basis to be understood in the obvious sense. In the case where $\mathbf{G}$ is singular, we set by convention $w:=0$.

If $u$ satisfies a uniform bound $|u(y)| \leq b$ over $\Gamma$, where $b>0$ is known, we introduce the truncated leastsquares approximation

$$
\tilde{w}=T_{b}(w), \quad T_{b}(t):=\operatorname{sign}(t) \min \{b,|t|\},
$$

which we also denote by $\tilde{P}_{m}^{n} u$ in the noiseless case.

The analysis in $[8,20]$ investigates the minimal amount of sampling $n(m) \geq m$ that allows an accurate approximation of the unknown function $u$ by the random approximations $w$ or $\tilde{w}$. The accuracy here is to be understood in the sense of a comparison between the error $\|u-w\|$ and the best approximation error $e_{m}(u)$. This analysis is based on probabilistic estimates comparing the norm $\|\cdot\|$ and its empirical counterpart $\|\cdot\|_{n}$ uniformly over the space $V_{m}$. This comparison amounts in estimating the deviation of the random matrix $\mathbf{G}$ from its expectation $\mathbb{E}(\mathbf{G})=\mathbf{I}$, where $\mathbf{I}$ is the $m \times m$ identity matrix, since for $v \in V_{m}$ and $\mathbf{v}$ the vector representing $v$ in the basis $\mathcal{B}_{L}$, one has

$$
\|v\|_{n}^{2}=\mathbf{v}^{T} \mathbf{G v} \quad \text { and } \quad\|v\|^{2}=\mathbf{v}^{T} \mathbf{I} \mathbf{v}
$$

so that, for any $0<\delta<1$,

$$
\left|\left\|\mathbf{G}-\mathbf{I}\left|\left\|\leq \delta \Leftrightarrow\left|\|v\|_{n}^{2}-\|v\|^{2}\right| \leq \delta\right\| v \|^{2}, \quad v \in V_{m},\right.\right.\right.
$$

where ||$|\cdot|||$ denotes the spectral norm of a matrix. For this purpose, one introduces the quantity

$$
K\left(V_{m}\right):=\sup _{y \in \Gamma} \sum_{j=1}^{m}\left|L_{j}(y)\right|^{2} .
$$

One can easily check, using Cauchy-Schwarz inequality, that

$$
K\left(V_{m}\right)=\sup _{v \in V_{m},\|v\|=1}\|v\|_{L^{\infty}(\Gamma)}^{2},
$$


from which we deduce that $K\left(V_{m}\right)$ does not depend on the choice of the orthonormal basis $\mathcal{B}_{L}$ and only depends on $V_{m}$ and $\rho$. The quantity $K\left(V_{m}\right)$ is also a uniform bound on the Froebenius norm of the random matrix $R=\left(L_{j}(y) L_{k}(y)\right)_{j, k=1, \ldots, m}$ and therefore allows to bound the deviation of $\mathbf{G}$ which is its empirical average from its expectation $\mathbf{I}$, based on concentration inequalities for matrix valued random variables.

One main result in [8] is that for any $r>0$ and the number of samples $n$ large enough such that

$$
\frac{n}{\ln n} \geq \frac{K\left(V_{m}\right)}{\kappa}
$$

where $\kappa:=\frac{\zeta}{1+r}$ with $\zeta:=\frac{1-\ln 2}{2} \approx 0.15$, the deviation between $\mathbf{G}$ and $\mathbf{I}$ satisfies the probabilistic estimate

$$
\operatorname{Pr}\left\{\||\mathbf{G}-\mathbf{I}|\| \mid>\frac{1}{2}\right\} \leq 2 n^{-r}
$$

This estimate implies that with probability at least $1-2 n^{-r}$ the least square problem is stable: indeed, with at least this probability, one has

$$
\|\left|\mathbf{G}^{-1}\right||| \leq 2 \text { and }\|\mathbf{G} \mid\| \leq \frac{3}{2},
$$

and therefore, according to (2.11)

$$
|||J| \mid \leq \sqrt{\frac{3}{2}} n^{-1 / 2}
$$

Therefore it follows from (2.9) that

$$
\|w\|^{2} \leq 6\left(\frac{1}{n} \sum_{j=1}^{n}\left|z_{j}\right|^{2}\right),
$$

also meaning, in the noiseless case, that

$$
\left\|P_{m}^{n} u\right\|^{2} \leq 6\|u\|_{n}^{2}
$$

Using this result, the following quasi-optimality results are proved in [8] for the truncated least-square approximation

- In the noiseless model, if $u$ satisfies a uniform bound $b$ over $\Gamma$, then

$$
\mathbb{E}\left(\left\|u-\tilde{P}_{m}^{n} u\right\|^{2}\right) \leq(1+\epsilon(n)) e_{m}(u)^{2}+8 b^{2} n^{-r},
$$

where $\epsilon(n):=\frac{4 \kappa}{\ln (n)}$.

- In the stochastic noise model, if $u$ satisfies a uniform bound $b$ over $\Gamma$, then

$$
\mathbb{E}\left(\|u-\tilde{w}\|^{2}\right) \leq(1+2 \epsilon(n)) e_{m}(u)^{2}+8\left(b^{2} n^{-r}+\sigma^{2} \frac{m}{n}\right),
$$

where $\sigma^{2}:=\sup _{y \in \Gamma} \mathbb{E}\left(|\eta|^{2} \mid y\right)$ is the noise level.

The deterministic noise model is not treated in [8]. As already mention, this model is relevant to describe the discretization error, and we therefore provide here an analogous result in this case.

Theorem 2.1. For any $r>0$, if $n$ satisfies condition (2.19), and $u$ satisfies a uniform bound $b$ over $\Gamma$, then under the deterministic noise model

$$
\mathbb{E}\left(\|u-\tilde{w}\|^{2}\right) \leq(1+2 \epsilon(n)) e_{m}(u)^{2}+(8+2 \epsilon(n))\|\eta\|^{2}+8 b^{2} n^{-r} .
$$

If $\eta=0$, corresponding to the noiseless model, the factor 2 in from of $\epsilon(n)$ can be removed. 
Proof. It is quite similar to that of ([8], Thm. 3), and so we sketch it. Introducing the event $\Omega_{+}^{n}:=\{\|\mathbf{G}-\mathbf{I}\| \mid \leq$ $\left.\frac{1}{2}\right\}$ for which $\operatorname{Pr}\left(\Omega_{+}^{n}\right)>1-2 n^{-r}$ by $(2.20)$, we have

$$
\mathbb{E}\left(\|u-\tilde{w}\|^{2}\right) \leq \int_{\Omega_{+}^{n}}\|u-\tilde{w}\|^{2} d \rho^{n}+8 b^{2} n^{-r} \leq \int_{\Omega_{+}^{n}}\|u-w\|^{2} d \rho^{n}+8 b^{2} n^{-r} .
$$

In the event $\Omega_{+}^{n}$ we have

$$
\begin{aligned}
\|u-w\|^{2} & =\left\|u-P_{m} u+P_{m}^{n}\left(u-P_{m} u\right)+P_{m}^{n} u-w\right\|^{2} \\
& =\left\|u-P_{m} u\right\|^{2}+\left\|P_{m}^{n}\left(u-P_{m} u\right)+P_{m}^{n} u-w\right\|^{2} \\
& \leq e_{m}(u)^{2}+2\left\|P_{m}^{n} h\right\|^{2}+2\left\|P_{m}^{n} \eta\right\|^{2},
\end{aligned}
$$

where $h:=u-P_{m} u$ and $\eta$ is the noise function. It follows that

$$
\mathbb{E}\left(\|u-\tilde{w}\|^{2}\right) \leq e_{m}(u)^{2}+2 \mathbb{E}\left(\left\|P_{m}^{n} h\right\|^{2}+\left\|P_{m}^{n} \eta\right\|^{2}\right)+8 b^{2} n^{-r} .
$$

In the noiseless model, we have $\eta=0$ and the same computation thus leads to

$$
\mathbb{E}\left(\|u-\tilde{w}\|^{2}\right) \leq e_{m}(u)^{2}+\mathbb{E}\left(\left\|P_{m}^{n} h\right\|^{2}\right)+8 b^{2} n^{-r} .
$$

Now for any function $g$, we may write with $\mathbf{g}=\left(g\left(y^{j}\right)\right)_{j=1, \ldots, n}^{t}$,

$$
\begin{aligned}
\mathbb{E}\left(\left\|P_{m}^{n} g\right\|^{2}\right) & \leq 8 \mathbb{E}\left(\|\mathbf{J g}\|_{\ell^{2}}^{2}\right)=8 \mathbb{E}\left(\sum_{k=1}^{m}\left(\frac{1}{n} \sum_{j=1}^{n} g\left(y^{j}\right) L_{k}\left(y^{j}\right)\right)^{2}\right) \\
& =8 \sum_{k=1}^{m} \frac{1}{n^{2}}\left(\sum_{i=1}^{n} \mathbb{E}\left(g\left(y^{j}\right)^{2} L_{k}\left(y^{i}\right)^{2}\right)+\sum_{j \neq i} \mathbb{E}\left(g\left(y^{i}\right) g\left(y^{j}\right) L_{k}\left(y^{i}\right) L_{k}\left(y^{j}\right)\right)\right) \\
& =8 \sum_{k=1}^{m} \frac{1}{n^{2}}\left(n \mathbb{E}\left(g(y)^{2} L_{k}(y)^{2}\right)+n(n-1) \mathbb{E}\left(g(y) L_{k}(y)\right)^{2}\right) .
\end{aligned}
$$

In the case $g=h=u-P_{m} u$, the second term is null since $\mathbb{E}\left(g(y) L_{k}(y)\right)=\int_{\Gamma} g(y) L_{k}(y) d \rho$, and we thus find that

$$
\mathbb{E}\left(\left\|P_{m}^{n} h\right\|^{2}\right) \leq 8 \frac{K\left(V_{m}\right)}{n}\|h\|^{2} \leq 2 \epsilon(n) e_{m}(u)^{2} .
$$

In the case $g=\eta$, we find

$$
\mathbb{E}\left(\left\|P_{m}^{n} \eta\right\|^{2}\right) \leq 8\left(\frac{K\left(V_{m}\right)}{n}+1-\frac{1}{n}\right)\|\eta\|^{2} \leq(8+2 \epsilon(n))\|\eta\|^{2} .
$$

We conclude the proof by combining these estimates.

It is also desirable to estimate the error between $u$ and its estimator in probability rather than in expectation. In the following we give such an estimate, for the non-truncated estimator $w=P_{m}^{n} u$, however using the best approximation error in the uniform norm

$$
e_{m}(u)_{\infty}:=\inf _{v \in V_{m}}\|u-v\|_{L^{\infty}(\Gamma)},
$$

which is obviously larger than $e_{m}(u)$. A similar result was already proven in [20] in the particular case of discrete least squares on univariate polynomial spaces, and for the noiseless model. Here, we treat the more general deterministic noise model. 
Theorem 2.2. For any $r>0$, under condition (2.19), one has under the deterministic noise model,

$$
\operatorname{Pr}\left(\|u-w\| \geq(1+\sqrt{2}) e_{m}(u)_{\infty}+2 \sqrt{3}\|\eta\|_{L^{\infty}(\Gamma)}\right) \leq 2 n^{-r} .
$$

Proof. As in the proof of Theorem 2.1, we use the event $\Omega_{+}^{n}:=\left\{|||\mathbf{G}-\mathbf{I}||| \leq \frac{1}{2}\right\}$, which satisfies $\operatorname{Pr}\left(\Omega_{+}^{n}\right) \geq$ $1-2 n^{-r}$. Given any draw in $\Omega_{+}^{n}$, we have for any $v \in V_{m}$

$$
\|u-w\| \leq\|u-v\|+\left\|v-P_{m}^{n} u\right\|+\left\|P_{m}^{n} \eta\right\| \leq\|u-v\|+\sqrt{2}\left\|v-P_{m}^{n} u\right\|_{n}+2 \sqrt{3}\|\eta\|_{n},
$$

where we have used (2.16) and (2.24). Since $\|u-v\|_{n}^{2}=\left\|u-P_{m}^{n} u\right\|_{n}^{2}+\left\|P_{m}^{n} u-v\right\|_{n}^{2}$, we deduce

$$
\|u-w\| \leq\|u-v\|+\sqrt{2}\|u-v\|_{n}+2 \sqrt{3}\|\eta\|_{n} \leq(1+\sqrt{2})\|u-v\|_{\infty}+2 \sqrt{3}\|\eta\|_{L^{\infty}(\Gamma)},
$$

which completes the proof.

All these above results lead to the problem of understanding which minimal amount $n$ of samples ensures the validity of condition (2.19). In the one-dimensional case $d=1$, with $V_{m}=\mathbb{P}_{m-1}$ being the space of polynomials of degree less or equal to $m-1$ and $\rho$ being the uniform measure over $\Gamma=[-1,1]$, elementary computations using the Legendre polynomials show that $K\left(V_{m}\right)=m^{2}$ and therefore (2.19) holds for $\frac{n}{\ln n} \sim m^{2}$, meaning that $n$ scales like $m^{2}$ up to a logarithmic factor. This relation between $n$ and $m$ was also obtained in [20] to establish estimates for the the discrete least-squares error in probability, however, by different arguments which are more tied to the use of univariate polynomials and the uniform measure. The next section discusses the implications of condition (2.19) for the multivariate polynomial spaces $\mathbb{P}_{\Lambda}$.

\section{LEAST-SQUARES APPROXIMATION WiTH MULTIVARIATE POLYNOMIALS}

In this section, we investigate the implications of the condition (2.19) in the setting of multivariate polynomial spaces $\mathbb{P}_{\Lambda}$. We consider the domain $\Gamma:=[-1,1]^{d}$ with $d \in \mathbb{N}$ and the uniform measure $\rho$ over $\Gamma$, i.e.

$$
\mathrm{d} \rho:=\otimes_{j=1}^{d} \frac{\mathrm{d} y_{j}}{2} .
$$

We may also consider the case $\Gamma:=[-1,1]^{\mathbb{N}}$ for which $d=+\infty$ and $\rho$ is the uniform measure defined over $\Gamma$ in the usual manner.

We use the notations $L^{2}(\Gamma, \rho),\langle\cdot, \cdot\rangle$ and $\|\cdot\|$ of the previous section and denote $\mathcal{F}$ the set of multi-indices in the cases $d<+\infty$ and $d=+\infty$ as explained in the introduction. Given $\Lambda$ a finite subset of $\mathcal{F}, u$ an unknown real valued function, and $\left(z^{i}\right)_{i=1, \ldots, n}$ noiseless or noisy observations of $u$ at the points $\left(y^{i}\right)_{i=1, \ldots, n}$ where the $y^{i}$ are i.i.d. random variables distributed according to $\rho$, we introduce the polynomial discrete least-squares approximation

$$
w:=\underset{v \in \mathbb{P}_{\Lambda}}{\operatorname{argmin}} \sum_{i=1}^{n}\left|z^{i}-v\left(y^{i}\right)\right|^{2},
$$

where the polynomial space $\mathbb{P}_{\Lambda}$ is defined as in (1.6). In order to study the optimality of the least-squares approximation, we need to investigate the growth of the quantity $K\left(V_{m}\right)$ introduced in (2.18) with $V_{m}=\mathbb{P}_{\Lambda}$. We shall show that, under the minimal requirement that the index set $\Lambda$ is downward closed, we have as in the one-dimensional case that $K\left(\mathbb{P}_{\Lambda}\right) \leq(\# \Lambda)^{2}$.

We introduce $\left(L_{k}\right)_{k \geq 0}$ the univariate Legendre polynomials normalized according to

$$
\int_{-1}^{1}\left|L_{k}(t)\right|^{2} \frac{\mathrm{d} t}{2}=1,
$$


and introduce $\left(L_{\nu}\right)_{\nu \in \mathcal{F}}$ the multivariate Legendre polynomials defined by

$$
L_{\nu}(y):=\prod_{j=1}^{d} L_{\nu_{j}}\left(y_{j}\right)
$$

The family $\left(L_{\nu}\right)_{\nu \in \mathcal{F}}$ is an orthonormal basis of the space $L^{2}(\Gamma, \rho)$. Using the remarks on lower sets given in the introduction, one has that $\left(L_{\nu}\right)_{\nu \in \Lambda}$ is an orthonormal basis of $\mathbb{P}_{\Lambda}$ if the index set $\Lambda$ is downward closed. Therefore, the multivariate extension of (2.17) reads

$$
K_{L}\left(\mathbb{P}_{\Lambda}\right):=\sup _{y \in \Gamma} \sum_{\nu \in \Lambda}\left|L_{\nu}(y)\right|^{2}=\sum_{\nu \in \Lambda}\left\|L_{\nu}\right\|_{L^{\infty}(\Gamma)}^{2}
$$

with the latter equality being valid since all the Legendre polynomials achieve their maximum on the boundary of $\Gamma$. Here, we use the subscript "L" to refer to the use of the uniform measure and therefore of mutivariate Legendre polynomials. To lighten the notation, in the following we shorten $K_{L}\left(\mathbb{P}_{\Lambda}\right)$ to $K_{L}(\Lambda)$. Since the univariate Legendre polynomials satisfy $\left\|L_{k}\right\|_{L^{\infty}([-1,1])}=\sqrt{2 k+1}$, then

$$
K_{L}(\Lambda)=\sum_{\nu \in \Lambda} \prod_{j}\left(2 \nu_{j}+1\right)
$$

Theses quantities have already been studied in [6] and proved to have moderate growth for finite lower sets. To keep our document self contained, we recall the result of [6] with its proof in the case $d=+\infty$. The case $d<+\infty$ is a straightforward consequence.

Lemma 3.1. For any finite lower set $\Lambda \subset \mathcal{F}$, the quantity $K_{L}(\Lambda)$ satisfies

$$
\#(\Lambda) \leq K_{L}(\Lambda) \leq(\#(\Lambda))^{2} .
$$

Proof. The first inequality is obvious. To prove the second inequality, we use induction on $n_{\Lambda}:=\#(\Lambda) \geq 1$. When $n_{\Lambda}=1$, then $\Lambda=\left\{0_{\mathcal{F}}\right\}$ and an equality holds. Let $n \geq 1$ and let $\Lambda$ denote a lower set with $n_{\Lambda}=n+1$. Without loss of generality, we suppose that $\nu_{1} \neq 0$ for some $\nu \in \Lambda$. We introduce the index sets

$$
\Lambda_{k}:=\{\hat{\nu} \in \mathcal{F}:(k, \hat{\nu}) \in \Lambda\}, \quad k \geq 0 .
$$

Here $(k, \hat{\nu})$ denote the multi-index $\left(k, \hat{\nu}_{1}, \hat{\nu}_{2}, \ldots\right)$. Since $\Lambda$ is downward closed and finite, then it is easy to check that the sets $\Lambda_{k}$ are finite, downward closed (when not empty) and satisfy

$$
\ldots \subset \Lambda_{k} \subset \ldots \subset \Lambda_{1} \subset \Lambda_{0} .
$$

Let us also remark that there exists $0 \leq J \leq n_{\Lambda}$ such that $\Lambda_{k}=\emptyset$ for any $k>J$ and that $\#\left(\Lambda_{0}\right) \leq n_{\Lambda}-1=n$ since $\nu_{1} \neq 0$ for some $\nu \in \Lambda$. Therefore the induction hypothesis applied to the sets $\Lambda_{k}$, implies

$$
K_{L}(\Lambda)=\sum_{k=0}^{J}(2 k+1) K_{L}\left(\Lambda_{k}\right) \leq \sum_{k=0}^{J}(2 k+1)\left(\#\left(\Lambda_{k}\right)\right)^{2} .
$$

Now, by the nestedness of the sets $\Lambda_{k}$, we have

$$
k\left(\#\left(\Lambda_{k}\right)\right)^{2} \leq \#\left(\Lambda_{k}\right) \#\left(\Lambda_{0}\right)+\ldots+\#\left(\Lambda_{k}\right) \#\left(\Lambda_{k-1}\right), \quad 1 \leq k \leq J .
$$

Therefore

$$
K_{L}(\Lambda) \leq \sum_{k=0}^{J}\left(\#\left(\Lambda_{k}\right)\right)^{2}+2 \sum_{k=1}^{J} \sum_{k^{\prime}=0}^{k-1} \#\left(\Lambda_{k}\right) \#\left(\Lambda_{k^{\prime}}\right)=\left(\sum_{k=0}^{J} \#\left(\Lambda_{k}\right)\right)^{2} .
$$

Since $\#(\Lambda)=\sum_{k=0}^{J} \#\left(\Lambda_{k}\right)$, we conclude the proof. 
The previous bound is valid for any lower set independently of its shape. In addition, the inequality is sharp, in the sense that the equality holds for certain types of lower sets. Indeed, given $\nu \in \mathcal{F}$ supported in $\{1, \ldots, J\}$ and considering the rectangle index set

$$
\mathcal{R}_{\nu}:=\{\mu \in \mathcal{F}: \mu \leq \nu\},
$$

one has

$$
K_{L}\left(\mathcal{R}_{\nu}\right)=\sum_{\mu \leq \nu} \prod_{1 \leq j \leq J}\left(2 \mu_{j}+1\right)=\prod_{1 \leq j \leq J} \sum_{\mu_{j} \leq \nu_{j}}\left(2 \mu_{j}+1\right)=\prod_{1 \leq j \leq J}\left(\nu_{j}+1\right)^{2}=\left(\#\left(\mathcal{R}_{\nu}\right)\right)^{2}
$$

However, we expect this bound to be pessimistic for lower sets that have shapes very different from rectangles. For instance, let $k \geq 1$ and consider the lower set

$$
S_{k, d}:=\left\{\nu \in \mathbb{N}_{0}^{d}:|\nu| \leq k\right\}
$$

where $|\nu|:=\sum_{j=1}^{d} \nu_{j}$, associated with the polynomial space $\mathbb{P}_{S_{k, d}}$ of total degree (TD) $k$ in dimension $d$.

By the inequality between the arithmetic and geometric means, one has for any $\nu \in S_{k, d}$

$$
\prod_{1 \leq j \leq d}\left(2 \nu_{j}+1\right) \leq\left(\frac{1}{d} \sum_{1 \leq j \leq d}\left(2 \nu_{j}+1\right)\right)^{d}=\left(\frac{2|\nu|}{d}+1\right)^{d} \leq\left(\frac{2 k}{d}+1\right)^{d} .
$$

Therefore (see also [18], Chaps. 2 and 3)

$$
K_{L}\left(S_{k, d}\right) \leq\left(\frac{2 k}{d}+1\right)^{d} \#\left(S_{k, d}\right)
$$

and $\left(\frac{2 k}{d}+1\right)^{d}$ is very small compared to $\#\left(S_{k, d}\right)=\left(\begin{array}{c}d+k \\ k\end{array}\right)$ for large values of $d$. On Figure 1, we provide a comparison between \#( $\left.S_{k, d}\right), K_{L}\left(S_{k, d}\right)$ and $\left(\#\left(S_{k, d}\right)\right)^{2}$ for various dimensions.

It is interesting to see if the estimates on the quantity $K\left(\mathbb{P}_{\Lambda}\right)$ can be improved when using other standard probability measures over $\Gamma$. In what follows, we study this quantity when the measure $\rho$ is the tensorized Chebyshev measure, i.e.

$$
\mathrm{d} \rho:=\otimes_{j=1}^{d} \varrho\left(y_{j}\right) \mathrm{d} y_{j}, \quad \text { with } \quad \varrho(t):=\frac{1}{\pi} \frac{1}{\sqrt{1-t^{2}}} .
$$

Using in this case the notation $K_{T}(\Lambda)=K\left(\mathbb{P}_{\Lambda}\right)$, we have

$$
K_{T}(\Lambda):=\left\|\sum_{\nu \in \Lambda}\left|T_{\nu}\right|^{2}\right\|_{L^{\infty}(\Gamma)}=\sum_{\nu \in \Lambda}\left\|T_{\nu}\right\|_{L^{\infty}(\Gamma)}^{2}
$$

where $T_{\nu}(y)=\prod_{j \geq 1} T_{\nu_{j}}\left(y_{j}\right)$ is the tensorization of the Chebyshev polynomials $\left(T_{k}\right)_{k \geq 0}$ normalized according to

$$
\int_{-1}^{1}\left|T_{k}(t)\right|^{2} \varrho(t) \mathrm{d} t=1
$$

It is easily checked that these polynomials are related to the classical Chebyshev polynomials of the first kind by $T_{k}(\cos \theta)=\sqrt{2} \cos (k \theta)$ for any $k \geq 1$ and $T_{0}=1$. It follows that

$$
K_{T}(\Lambda)=\sum_{\nu \in \Lambda} 2^{\#(\operatorname{supp}(\nu))}
$$



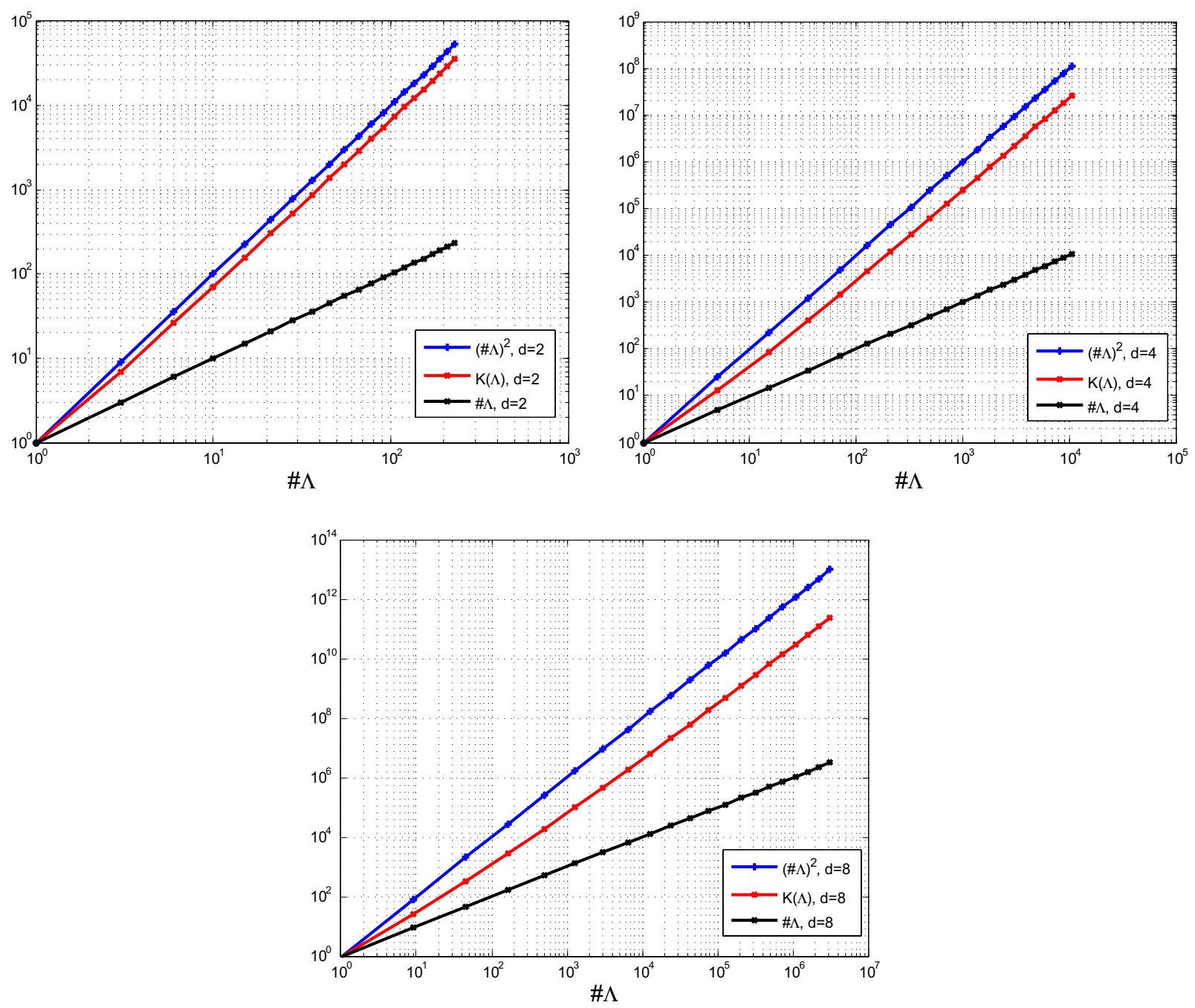

Figure 1. Comparison between $\#(\Lambda), K_{L}(\Lambda)$ and $(\#(\Lambda))^{2}$ in the case where $\Lambda=S_{k, d}$ (see Eq. (3.15)). Left: $d=2$. Center: $d=4$. Right: $d=8$.

where $\operatorname{supp}(\nu):=\left\{1 \leq j \leq d: \nu_{j} \neq 0\right\}$ is the support of $\nu \in \mathcal{F}$. Given $\nu$ in $\Lambda$, with $\Lambda$ being a lower set, the multi-index $\mu$ that has the same support as $\nu$ and has entries 1 satisfies $\mu \leq \nu$, so that $\mu \in \Lambda$ and $\mathcal{R}_{\mu} \subset \Lambda$. This implies that $2^{\#(\operatorname{supp}(\nu))}=\#\left(\mathcal{R}_{\mu}\right) \leq \#(\Lambda)$. Therefore we obtain

$$
K_{T}(\Lambda) \leq(\#(\Lambda))^{2},
$$

which is the same bound as for the uniform measure.

Sharper bounds can be established by a finer analysis. We first prove an elementary lemma.

Proposition 3.2. For any real positive numbers $a_{0} \geq a_{1} \geq \ldots \geq a_{k}$ and any $\alpha \geq \frac{\ln 3}{\ln 2}$, one has

$$
a_{0}^{\alpha}+2\left(a_{1}^{\alpha}+\ldots+a_{k}^{\alpha}\right) \leq\left(a_{0}+\ldots+a_{k}\right)^{\alpha} .
$$


Proof. We use induction on $k$. For $k=0$, equality holds in (3.23). For $k=1$, since the function $x \mapsto\left(x+a_{1}\right)^{\alpha}-x^{\alpha}$ is increasing in $\left[a_{1},+\infty\left[\right.\right.$ then its value at $a_{0}$ is greater than its value at $a_{1}$, that is

$$
2 a_{1}^{\alpha} \leq\left(2^{\alpha}-1\right) a_{1}^{\alpha} \leq\left(a_{0}+a_{1}\right)^{\alpha}-a_{0}^{\alpha}
$$

where we have used $2^{\alpha}>3$. Now let $k \geq 1$ and $a_{0} \geq a_{1} \geq \ldots \geq a_{k+1}$ be real positive numbers. By the induction hypothesis at steps 1 and $k$, we infer

$$
\begin{aligned}
\left(a_{0}+\ldots+a_{k+1}\right)^{\alpha} & =\left(\left(a_{0}+\ldots+a_{k}\right)+a_{k+1}\right)^{\alpha} \\
& \geq\left(a_{0}+\ldots+a_{k}\right)^{\alpha}+2 a_{k+1}^{\alpha} \\
& \geq a_{0}^{\alpha}+2\left(a_{1}^{\alpha} \ldots+a_{k}^{\alpha}\right)+2 a_{k+1}^{\alpha} \\
& =a_{0}^{\alpha}+2\left(a_{1}^{\alpha} \ldots+a_{k+1}^{\alpha}\right) .
\end{aligned}
$$

The proof is then complete.

Lemma 3.3. For any lower set $\Lambda \subset \mathcal{F}$, the quantity $K_{T}(\Lambda)$ satisfies

$$
K_{T}(\Lambda) \leq(\#(\Lambda))^{\beta}, \quad \text { with } \quad \beta=\frac{\ln 3}{\ln 2} .
$$

Proof. We use induction on $n_{\Lambda}:=\#(\Lambda)$. When $n_{\Lambda}=1$, then $\Lambda=\left\{0_{\mathcal{F}}\right\}$ and an equality holds. Let $n \geq 1$ and $\Lambda$ denote a lower set with $n_{\Lambda}=n+1$. Without loss of generality, we suppose that $\nu_{1} \neq 0$ for some $\nu \in \Lambda$. Defining $J \geq 0$ and the sets $\Lambda_{k}$ as in the proof of Lemma 3.1 and using the induction hypothesis with these sets, we obtain

$$
K_{T}(\Lambda)=\sum_{k=0}^{J} \gamma(k) K_{T}\left(\Lambda_{k}\right) \leq \sum_{k=0}^{J} \gamma(k)\left(\#\left(\Lambda_{k}\right)\right)^{\frac{\ln 3}{\ln 2}}
$$

where $\gamma$ is defined by $\gamma(0)=1$ and $\gamma(k)=2$ for $k \geq 1$. Using (3.23), we infer

$$
K_{T}(\Lambda) \leq\left(\#\left(\Lambda_{0}\right)\right)^{\frac{\ln 3}{\ln 2}}+2 \sum_{k=1}^{J}\left(\#\left(\Lambda_{k}\right)\right)^{\frac{\ln 3}{\ln 2}} \leq\left(\#\left(\Lambda_{0}\right)+\#\left(\Lambda_{1}\right)+\ldots+\#\left(\Lambda_{J}\right)\right)^{\frac{\ln 3}{\ln 2}}=(\#(\Lambda))^{\frac{\ln 3}{\ln 2}} .
$$

The proof is then complete.

The bound (3.26) is sharp for certain type of lower sets. For instance if $\nu$ is the multi-index such that $\nu_{1}=\ldots=\nu_{J}=1$ and $\nu_{j}=0$ for $j>J$, then

$$
K_{T}\left(\mathcal{R}_{\nu}\right)=\sum_{\mu \leq \nu} 2^{\#(\operatorname{supp}(\mu))}=\sum_{\mu \leq \nu} 2^{\mu_{1}+\ldots+\mu_{J}}=\prod_{j=1}^{J}(1+2)=3^{J}=\left(2^{J}\right)^{\beta}=\left(\#\left(\mathcal{R}_{\nu}\right)\right)^{\beta} .
$$

In the case of finite dimension $d<+\infty$, the following bound can be easily obtained from the result of Lemma 3.3:

$$
K_{T}(\Lambda) \leq \min \left\{(\#(\Lambda))^{\frac{\ln 3}{\ln 2}}, 2^{d} \#(\Lambda)\right\} .
$$

Let us mention that similar algebraic bounds can also be obtained when the measure $\rho$ is of the more general type

$$
\mathrm{d} \rho:=\otimes_{j=1}^{\mathrm{d}} \varrho\left(y_{j}\right) d y_{j}, \quad \varrho(t)=\frac{(1-t)^{\alpha_{1}}(1+t)^{\alpha_{2}}}{\int_{-1}^{1}(1-t)^{\alpha_{1}}(1+t)^{\alpha_{2}} \mathrm{~d} t}, \quad \alpha_{1}, \alpha_{2}>-1,
$$

that is, the tensorization of the $\beta\left(\alpha_{1}, \alpha_{2}\right)$ measure. In this case, the relevant quantity is

$$
K_{J}(\Lambda)=\left\|\sum_{\nu \in \Lambda}\left|J_{\nu}^{\alpha_{1}, \alpha_{2}}\right|^{2}\right\|_{L^{\infty}(\Gamma)}
$$

where $J_{\nu}^{\alpha_{1}, \alpha_{2}}$ are the tensorized Jacobi polynomials. For this quantity, the following has been proven in [19], in the case where $\alpha_{1}, \alpha_{2}$ are natural exponents. 
Lemma 3.4. For any lower set $\Lambda \subset \mathcal{F}$, the quantity $K_{J}(\Lambda)$ with Jacobi polynomials $\left(J_{\nu}^{\alpha_{1}, \alpha_{2}}\right)_{\nu \in \Lambda}$ and $\alpha_{1}, \alpha_{2} \in$ $\mathbb{N}_{0}$ satisfies

$$
K_{J}(\Lambda) \leq(\#(\Lambda))^{2 \max \left\{\alpha_{1}, \alpha_{2}\right\}+2} .
$$

Note that this result includes the estimate $K_{L}(\Lambda) \leq(\#(\Lambda))^{2}$ as the particular case $\alpha_{1}=\alpha_{2}=0$. Combining the estimates on $K_{T}(\Lambda)$ and $K_{J}(\Lambda)$, with the results stated in the previous section, we arrive at our main theorem for multivariate polynomial least-squares.

Theorem 3.5. For any $r>0$, given a finite lower set $\Lambda$, if the measure $\rho$ is the tensorized beta $\left(\alpha_{1}, \alpha_{2}\right)$ with $\alpha_{1}, \alpha_{2} \in \mathbb{N}_{0}$ and

$$
\frac{n}{\ln n} \geq \frac{1+r}{\zeta}(\#(\Lambda))^{2 \max \left\{\alpha_{1}, \alpha_{2}\right\}+2}
$$

or, if the measure $\rho$ is the tensorized Chebyshev measure and

$$
\frac{n}{\ln n} \geq \frac{1+r}{\zeta}(\#(\Lambda))^{\frac{\ln 3}{\ln 2}}
$$

then the following holds true:

(i) The deviation between $\mathbf{G}$ and $\mathbf{I}$ satisfies

$$
\operatorname{Pr}\left\{|||\mathbf{G}-\mathbf{I}| \| \mid>\frac{1}{2}\right\} \leq 2 n^{-r} .
$$

(ii) Under the deterministic noise model, if u satisfies a uniform bound b over $\Gamma$, then one has the estimate in expectation

$$
\mathbb{E}\left(\|u-\tilde{w}\|^{2}\right) \leq(1+2 \epsilon(n)) e_{m}(u)^{2}+(8+2 \epsilon(n))\|\eta\|^{2}+8 b^{2} n^{-r},
$$

where the factor 2 in front of $\epsilon(n)$ can be removed when $\eta=0$.

(iii) Under the same deterministic noise model, one also has the estimate in probability

$$
\operatorname{Pr}\left(\|u-w\| \geq(1+\sqrt{2}) e_{m}(u)_{\infty}+2 \sqrt{3}\|\eta\|_{L^{\infty}}\right) \leq 2 n^{-r} .
$$

\section{Discrete least-Squares approximation of Hilbert space-Valued functions}

In Sections 2 and 3, the functions that we propose to approximate using the least-squares method are real valued. Motivated by the application to parametric PDEs, we investigate the applicability of the least-squares method in the approximation of $X$-valued functions, with $X$ being any Hilbert space. Similar to Section 2, we work in the abstract setting of a probability space $(\Gamma, \Sigma, \rho)$. We study the least-squares approximation of functions $u$ belonging to the Bochner space

$$
L^{2}(\Gamma, X, \rho):=\left\{u: \Gamma \rightarrow X,\|u\|:=\int_{\Gamma}\|u(y)\|_{X}^{2} \mathrm{~d} \rho(y)<+\infty\right\} .
$$

Therefore $L^{2}(\Gamma, X, \rho)=X \otimes L^{2}(\Gamma, \rho)$ and we are interested in the least-squares approximation in spaces of type $X \otimes V_{m}$, where $V_{m}$ is an $m$-dimensional subspace of $L^{2}(\Gamma, \rho)$. Given $u \in L^{2}(\Gamma, X, \rho)$ an unknown function and $\left(z^{i}\right)_{i=1, \ldots, n}$ noiseless or noisy observations of $u$ at the points $\left(y^{i}\right)_{i=1, \ldots, n}$ where the $y^{i}$ are i.i.d. random variables distributed according to $\rho$, we consider the discrete least-squares approximation

$$
w:=\underset{v \in X \otimes V_{m}}{\operatorname{argmin}} \sum_{i=1}^{n}\left\|z^{i}-v\left(y^{i}\right)\right\|_{X}^{2} .
$$

The purpose of this section is to briefly discuss the extension of the results from Section 2 to this framework. 
Let $\mathcal{B}_{L}$ be an orthonormal basis of the space $V_{m}$ with respect to the measure $\rho$ and consider the matrices $\mathbf{G}$ and $\mathbf{J}$ and the family $\mathcal{B}_{\pi} \subseteq V_{m}$ obtained from the basis $\mathcal{B}_{L}$ and the points $\left(y^{i}\right)_{i=1, \ldots, n}$ as in Section 2. When the matrix $\mathbf{G}$ is not singular, we claim that the solution to (4.2) has the same form

$$
\sum_{k=1}^{n} z^{k} \pi_{k}
$$

with $z^{k} \in X$ for all $k=1, \ldots, n$, as in the real-valued case. Indeed, for any $g \in X$, the real-valued function $w_{g}:=\sum_{k=1}^{n}\left\langle z^{k}, g\right\rangle \pi_{k} \in V_{m}$ is the solution to the least-squares problem

$$
w_{g}=\underset{h \in V_{m}}{\operatorname{argmin}} \sum_{i=1}^{n}\left|\left\langle z^{i}, g\right\rangle-h\left(y^{i}\right)\right|^{2},
$$

which implies the orthogonality relations

$$
\sum_{i=1}^{n}\left\langle\sum_{k=1}^{n} z^{k} \pi_{k}\left(y^{i}\right), g L_{j}\left(y^{i}\right)\right\rangle=\sum_{i=1}^{n}\left\langle z^{i}, g L_{j}\left(y^{i}\right)\right\rangle, \quad g \in X, j \in\{1, \ldots, m\},
$$

showing that $\sum_{k=1}^{n} z^{k} \pi_{k}$ is the solution to (4.2). When the matrix $\mathbf{G}$ is singular, the solution (4.2) is non-unique and we set by convention $w:=0$.

The explicit formula of the least-squares approximation (4.2) being established, we are interested in the stability and accuracy of the approximation. Similarly to the analysis in Section 2, we investigate the comparability over $X \otimes V_{m}$ of the norm $\|\cdot\|$ and its empirical counterpart $\|\cdot\|_{n}$ defined by

$$
\|v\|_{n}=\left(\frac{1}{n} \sum_{j=1}^{n}\left\|v\left(y^{j}\right)\right\|_{X}^{2}\right)^{\frac{1}{2}}, \quad v \in L^{2}(\Gamma, X, \rho) .
$$

It is easily checked that given $v:=\sum_{j=1}^{m} v_{j} L_{j} \in X \otimes V_{m}$, one has

$$
\|v\|_{n}^{2}-\|v\|^{2}=\sum_{i=1}^{m} \sum_{j=1}^{m}(\mathbf{G}-\mathbf{I})_{i j}\left\langle v_{i}, v_{j}\right\rangle_{X}=\langle\mathbf{v},(\mathbf{G}-\mathbf{I}) \mathbf{v}\rangle_{X^{m}}
$$

where $\mathbf{v}:=\left(v_{1}, \ldots, v_{m}\right)^{t} \in X^{m}$ and the matrix-vector product is defined as in the real case. Here the inner product $\langle\cdot, \cdot\rangle_{X^{m}}$ is the standard inner product over $X^{m}$ constructed from $\langle\cdot, \cdot\rangle_{X}$. Note that we have $\|v\|=\|\mathbf{v}\|_{X^{m}}$. We next observe that if $\mathbf{M}$ is an $m \times m$ real symmetric matrix, one has

$$
\sup _{\|\mathbf{v}\|_{X^{m}=1}}\left|\langle\mathbf{v}, \mathbf{M v}\rangle_{X^{m}}\right|=|\|\mathbf{M}\|| \mid
$$

where $\||\mathbf{M} \||$ is the spectral norm of $M$ (this is immediately checked by diagonalizing $M$ in an orthonormal basis). Therefore it holds that

$$
\left|\|v\|_{n}^{2}-\|v\|^{2}\right| \leq|| \mathbf{G}-\mathbf{I}|| \mid\|v\|^{2},
$$

and, similarly to the results discussed in Section 2, we find that under condition (2.19) the norm $\|\cdot\|$ and its counterpart $\|\cdot\|_{n}$ are equivalent over $X \otimes V_{m}$ with probability greater than $1-2 n^{-r}$, with

$$
\left|\|v\|_{n}^{2}-\|v\|^{2}\right| \leq \frac{1}{2}\|v\|^{2}
$$


Similar to real valued functions, we want to compare the accuracy of the least-squares approximation (4.2) with the error of best approximation in $L^{2}(\Gamma, X, \rho)$

$$
e_{m}(u):=\inf _{v \in X \otimes V_{m}}\|u-v\|=\left\|u-P_{m} u\right\|,
$$

where $P_{m}$ is the orthogonal projector onto $X \otimes V_{m}$.

We again use the notation $P_{m}^{n} u$ for the least-squares solution in the noiseless case. If $u$ satisfies a unifom bound $\|u(y)\|_{X} \leq b$ over $\Gamma$ where $b$ is known, we define the truncated least-squares approximation

$$
\tilde{w}=T_{b}(w),
$$

also denoted by $\tilde{P}_{m}^{n} u$ in the noiseless case, where $T_{b}$ is the trunction operator, now defined as follows

$$
T_{b}(v)=\left\{\begin{array}{l}
v \text { if }\|v\| \leq b, \\
\frac{v}{\|v\|} b \text { if }\|v\|>b .
\end{array}\right.
$$

Note that $T_{b}$ is the projection map onto the closed disc $\{\|v\| \leq b\}$ and is therefore Lipschitz continuous with constant equal to 1 .

With such definitions, the result of Theorem 2.1 remains valid for Hilbert space valued functions with the exact same proof as for real valued functions. Likewise, with

$$
e_{m}(u)_{\infty}=\inf _{v \in X \otimes V_{m}}\|u-v\|_{L^{\infty}(\Gamma, X)}
$$

Theorem 2.2 remains valid for Hilbert space valued functions with the exact same proof as for real valued functions. In turn, the approximation results listed in (i) and (ii) of Theorem 3.5 are also valid for multivariate polynomial least-squares applied to Hilbert space valued functions.

As a general example of application, consider the model stochastic elliptic boundary value problem (1.9) with a diffusion coefficient given by (1.10) and satisfying (1.11). As recalled in the introduction, if $\left(\left\|\psi_{j}\right\|_{L^{\infty}(D)}\right)_{j \geq 1} \in$ $\ell^{p}(\mathbb{N})$ for some $p<1$, then there exists a nested sequence of lower sets

$$
\Lambda_{1} \subset \Lambda_{2} \subset \ldots \subset \mathcal{F}, \quad \#\left(\Lambda_{m}\right)=m,
$$

such that with $X:=H_{0}^{1}(D)$ and $V_{m}:=\mathbb{P}_{\Lambda_{m}}$ one has

$$
e_{m}(u) \leq C m^{-s}, \quad s:=\frac{1}{p}-\frac{1}{2}>0 .
$$

Since the solution satisfies the uniform bound $\|u(y)\|_{X} \leq b:=\frac{\|f\|_{V^{*}}}{r}$, we can compute its trunctated leastsquares approximation $\tilde{P}_{m}^{n} u$ based on $n$ observations $u^{i}=u\left(y^{i}\right)$ where the $y^{i}$ are i.i.d. with respect to the uniform measure over $\Gamma:=[-1,1]^{\mathbb{N}}$. Combining Theorem 2.1 for the noiseless model and (3.7), it follows that

$$
\mathbb{E}\left(\left\|u-\tilde{P}_{m}^{n} u\right\|^{2}\right) \leq(1+\epsilon(n)) C^{2} m^{-2 s}+8 b^{2} n^{-r},
$$

provided that $\frac{n}{\ln n} \geq \frac{m^{2}}{\kappa}$ with $\kappa:=\frac{1-\ln 2}{2+2 r}$. In particular, taking $r=s$, we obtain the estimate

$$
\mathbb{E}\left(\left\|u-\tilde{P}_{m}^{n} u\right\|^{2}\right) \lesssim m^{-2 s} .
$$

Taking the minimal amount of sample $n$ such that $\frac{n}{\ln n} \geq \frac{m^{2}}{\kappa}$, this gives the convergence estimate

$$
\mathbb{E}\left(\left\|u-\tilde{P}_{m}^{n} u\right\|^{2}\right) \lesssim\left(\frac{n}{\ln n}\right)^{-s} .
$$


Remark 4.1. The error in the evalution of $u\left(y^{i}\right)$ due to space discretization can be taken into account in several ways. In the case where the space discretization is independent of the parameter $y$, for example if one uses the same finite element space $X_{h}$ independently of $y$, we may view the polynomial least squares approximation as the noiseless approximation $\tilde{P}_{m}^{n} u_{h}$ of the discrete solution map $y \mapsto u_{h}(y) \in X_{h}$. This allows to decompose the total error into

$$
\left\|u-\tilde{P}_{m}^{n} u_{h}\right\| \leq\left\|u_{h}-\tilde{P}_{m}^{n} u_{h}\right\|+\varepsilon_{\text {disc }},
$$

where the second term $\varepsilon_{\text {disc }}$ is a uniform bound on the space discretization error, and where similar convergence bounds to (4.18) can be obtained for the first term. An analogous approach was used in [5] for the analysis of polynomial approximation obtained by truncated Taylor series. However, in the more general case where the space discretization varies for different values of $y$, one cannot apply this strategy and a better adapted approach is to view the space discretization error as an additive deterministic noise in the observation model. Using Theorem 2.1 we then obtain the same estimate as (4.18) for the error $\|u-\tilde{w}\|$, where $\tilde{w}$ is the truncated polynomial least squares estimate based on the discretized solution intances, up to the addition of the uniform bound $\varepsilon_{\text {disc }}$ on the space discretization error. Both approaches therefore lead to the same type of estimate, but the second one applies to more general settings.

Remark 4.2. An analysis of the Chebyshev coefficients of $u$ reveals that the same approximation rate as (4.15) holds for the $L^{2}$ norm with respect to the tensorized Chebyshev measure. However, in view of (3.26), the condition between $m$ and $n$ is now $\frac{n}{\ln n} \geq \frac{m^{\beta}}{\kappa}$ with $\beta:=\frac{\ln 3}{\ln 2}$. It follows that the rate in (4.18) can be improved into

$$
\mathbb{E}\left(\left\|u-\tilde{P}_{m}^{n} u\right\|^{2}\right) \lesssim\left(\frac{n}{\ln n}\right)^{-\frac{2 \ln 3}{\ln 2} s},
$$

if we use samples $y^{i}$ that are i.i.d. with respect to the tensorized Chebyshev measure and if we use the $L^{2}$ error with respect to this measure. However, since the $L^{2}$-norm with Chebyshev weight controls the $L^{2}$-norm with the uniform weight, i.e. $\|u\|_{L_{u n i f}^{2}} \leq \sqrt{\pi / 2}\|u\|_{L_{\text {Cheb }}^{2}}$, estimate (4.20) holds also with $L^{2}$ norm with uniform weight.

\section{Application to elliptic PDEs with Random inclusions}

In this section, we focus on the subclass of stochastic PDEs (1.9) and (1.10) characterized by functions $\psi_{j}$ having nonoverlapping support. This situation allows to model, for instance, the diffusion process in a medium with nonoverlapping inclusions of random conductivity (see e.g. Fig. 2). We show in what follows that exponential bounds for the discrete least-squares approximation error in expectation can be obtained, in this case, however under a slightly more demanding condition $n \sim m^{2+1 / d}$ than what shown in the previous section for algebraic convergence rates. It has been shown in [4] that the solution map $u=u(y)$ admits a holomorphic complex continuation $u^{*}: \mathbb{C}^{d} \rightarrow H_{0}^{1}(\Omega)$ in a polyellipse $\mathcal{E}_{d} \subset \mathbb{C}^{d}$, where $\mathcal{E}_{d}=\mathcal{E}_{d}\left(g_{1}, \ldots, g_{d}\right):=\prod_{n=1}^{d} \mathcal{E}_{n, g_{n}}$ and $\mathcal{E}_{n, g_{n}}:=\left\{z \in \mathbb{C}, \operatorname{Re}(z)=\cosh \left(2 g_{n}\right) \cos (\theta), \operatorname{Im}=\sinh \left(2 g_{n}\right) \sin (\theta), \theta \in[0,2 \pi)\right\}$ and such that $B_{u}:=\sup _{z \in \mathcal{E}_{d}}\left\|u^{*}(z)\right\|_{H_{0}^{1}(\Omega)} \leq+\infty$. In this case, a priori estimates on the Legendre coefficients have been obtained e.g. in [4] and have been shown numerically to be quite sharp. They read:

$$
\left\|u_{\nu}\right\|_{X} \leq C \prod_{j=1}^{d} \exp \left\{-\nu_{j} g_{j}\right\}, \quad \forall \nu=\left(\nu_{1}, \ldots, \nu_{d}\right) \in \mathbb{N}_{0}^{d}
$$

with $X=H_{0}^{1}(D)$, where $C$ depends on $d,\left(g_{1}, \ldots, g_{d}\right)$ and $B_{u}$. Explicit expressions for the constant $C$ can be found in ([4], Cor. 9 (with $\epsilon=1 / 2)$ ). In practice, the coefficients $\left(g_{j}\right)_{1 \leq j \leq d}$ can be estimated through an a posteriori procedure, that requires to solve only "one-dimensional" problems, i.e. analyzing the convergence when considering one random variable at a time and freezing all other variables to their expected value. As a consequence, quasi-optimal index sets associated with the problems in the aforementioned class are of the form 


$$
\Lambda_{\mathrm{w}}=\left\{\nu \in \mathbb{N}_{0}^{d}: \sum_{j=1}^{d} g_{j} \nu_{j} \leq w\right\}, \quad w=0,1, \ldots
$$

and correspond to anisotropic total degree spaces, i.e. the anisotropic variants of (3.15). Analogous estimates, showing the optimality of the total degree space, have been presented in [5].

In the remaining discussion, we consider the simple isotropic case where $g_{j}=g$ for all $j=1, \ldots, d$. Observe that this analysis can also be taken as a (crude) upper bound for the anisotropic case by taking $g=\min _{j} g_{j}$. For convenience we introduce the following quantity:

$$
\phi:=\frac{C^{2}}{\left(1-e^{-g}\right)^{d}} \exp \left\{\frac{2 \operatorname{egd}\left(1-e^{-1}\right)}{5}\right\} .
$$

Then, from the results in [4] the following estimate of the exact $L^{2}$ projection error holds.

Lemma 5.1. In the isotropic case, i.e. $g_{j}=g$ for all $j=1, \ldots, d$, the following estimate holds for the error of the $L^{2}$ projection $P_{m}$ on the quasi-optimal lower sets $(5.1)$ with $\#(\Lambda)=m$ :

$$
\left\|u-P_{m} u\right\|^{2} \leq \phi \exp \left\{-g d e^{-1} m^{1 / d}\right\}
$$

for any $m>(2 e / 5)^{d}$.

Proof. The following estimate has been obtained in ([4], Thm. 22):

$$
\left\|u-P_{m} u\right\|^{2} \leq \frac{C^{2}}{\left(1-e^{-g}\right)^{d}} \exp \left\{-g d e^{-1} \ln \left((1-\xi(m))^{-1}\right) m^{1 / d}\right\},
$$

with

$$
\xi(m):=\left(1-e^{-1}\right)\left(1-\frac{2 e}{5 m^{1 / d}}\right) .
$$

Observe that we have omitted the factor $C_{\text {opt }}$ appearing in the mentioned theorem, as we look at the $L^{2}$ projection error and not at the Galerkin error. If $(2 e / 5)^{d}<m$ then $(1-\xi(m))<1$, and the exponential term on the right-hand side in (5.4) can be bounded as

$$
\begin{aligned}
(1-\xi(m))^{g d e^{-1} m^{1 / 2}} & =\left(e^{-1}+\frac{2 e\left(1-e^{-1}\right)}{5 m^{1 / d}}\right)^{g d e^{-1} m^{1 / d}} \\
& =\exp \left\{-g d e^{-1} m^{1 / d}\right\}\left(1+\frac{2 e^{2}\left(1-e^{-1}\right)}{5 m^{1 / d}}\right)^{g d e^{-1} m^{1 / d}} \\
& <\exp \left\{\frac{2 e\left(1-e^{-1}\right)}{5} g d\right\} \exp \left\{-g d e^{-1} m^{1 / d}\right\},
\end{aligned}
$$

and using the definition of $\phi$ we finally obtain the thesis.

Using the previous result and (3.36), we can now analyze the convergence in expectation of the discrete least-squares approximation based on $n$ noiseless observations $u^{i}=u\left(y^{i}\right)$ where $\left(y^{i}\right)_{1 \geq i \geq n}$ are i.i.d. with respect to the uniform measure over $\Gamma:=[-1,1]^{d}$. In particular, the parameter $r$ appearing in (3.36) has to be properly chosen as a function of $n$ to balance the two error terms in (3.36). This leads to a condition $n \sim m^{2+1 / d}$. 
Theorem 5.2. In the aforementioned PDE model class, when the number of points $n$ distributed according to the uniform measure is related to the cardinality $m$ of the polynomial space by the relation

$$
n \geq \frac{2 g d}{e \zeta} m^{2+\frac{1}{d}}, \quad \text { with } \zeta=\frac{1-\ln 2}{2},
$$

then the convergence rate of the discrete least-squares approximation with an optimal choice of the polynomial space satisfies

$$
\mathbb{E}\left(\left\|u-\tilde{P}_{m}^{n} u\right\|^{2}\right) \leq\left((1+\epsilon(n)) \tilde{\phi}+8 b^{2}\right) \exp \left\{-\left(\frac{\left(g d e^{-1}\right)^{2 d} \zeta n}{2}\right)^{\frac{1}{2 d+1}}\right\},
$$

with $\tilde{\phi}:=\phi \exp \left\{g d e^{-1}\right\}$.

Proof. We start from (3.36) in the noiseless case $\eta=0$, and recall that, in the case of uniform measure and polynomial spaces with downward closed index sets $\Lambda$, the cardinality of the set $m=\# \Lambda$ should satisfy (3.33) (for $\alpha_{1}=\alpha_{2}=0$ ). For a given $n$ we now take

$$
m=\left\lfloor\left(\frac{\zeta}{2 r} \frac{n}{\ln n}\right)^{\frac{1}{2}}\right\rfloor
$$

which satisfies (3.33) for any $r \geq 1$. To achieve the fastest convergence, the value of $r$ can be optimally selected as a function of the remaining parameters $n, \zeta, g$ and $d$. Replacing (5.9) in the right-hand side of (5.3), we obtain for the best $L^{2}$ approximation error and any $r \geq 1$

$$
\begin{aligned}
\left\|u-P_{m} u\right\|^{2} & \leq \phi \exp \left\{-\frac{g d}{e}\left[\left(\frac{\zeta n}{2 r \ln n}\right)^{\frac{1}{2}}\right]^{\frac{1}{d}}\right\} \\
& \leq \phi \exp \left\{-\frac{g d}{e}\left(\left(\frac{\zeta n}{2 r \ln n}\right)^{\frac{1}{2}}-1\right)^{\frac{1}{d}}\right\} \\
& \leq \tilde{\phi} \exp \left\{-\frac{g d}{e}\left(\frac{\zeta n}{2 r \ln n}\right)^{\frac{1}{2 d}}\right\} .
\end{aligned}
$$

Since we have embedded the stability condition (3.33) as a constraint, we can apply (3.36) in the noiseless case $\eta=0$ and use (5.10) to bound the best approximation error. Hence we obtain

$$
\mathbb{E}\left(\left\|u-\tilde{P}_{m}^{n} u\right\|^{2}\right) \leq(1+\epsilon(n)) \tilde{\phi} \exp \left\{-\frac{g d}{e}\left(\frac{\zeta n}{2 r \ln n}\right)^{\frac{1}{2 d}}\right\}+8 b^{2} \exp \{-r \ln n\} .
$$

Now we can choose $r$ as a function of $n$ and $d$ such that the exponents of the two exponential terms in (5.11) are equal, i.e.

$$
r=\frac{1}{\ln n}\left(\frac{\left(g d e^{-1}\right)^{2 d} \zeta n}{2}\right)^{\frac{1}{2 d+1}}
$$

Finally, substituting this expression of $r$ into (5.11) gives (5.8), which holds under condition (5.7) that is obtained after replacing (5.12) into (5.9). 


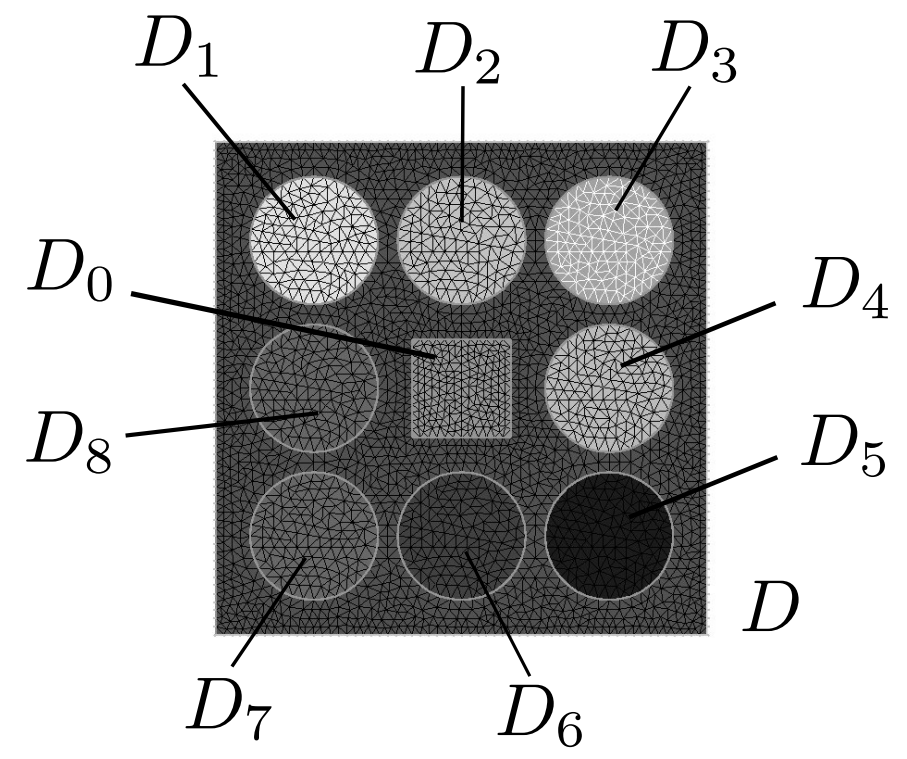

Figure 2. Mesh discretization and geometries of the inclusions. The domain $D$ is the unitary square. The inner square is named $D_{0}$, the eight circular inclusions are $D_{1}, \ldots, D_{8}$.

In (5.8) we observe that the error converges to zero sub-exponentially as $\exp \left\{-\alpha n^{\frac{1}{2 d+1}}\right\}$ with $\alpha:=$ $(d g / e)^{\frac{2 d}{2 d+1}}(\zeta / 2)^{\frac{1}{2 d+1}}$. The dimension $d$ appears both in the factor $\alpha$, favoring the convergence, and in the exponent of $n^{\frac{1}{2 d+1}}$, slowing down the convergence. A comparison with the convergence rate of the best $m$-term exact $L^{2}$ projection reveals that, to achieve the optimal exponential convergence rate $\mathcal{O}\left(\exp \left\{-g d e^{-1} m^{1 / d}\right\}\right)$ in terms of the dimension of the polynomial space, one has to use a number of observations that scales as $n \sim m^{2+1 / d}$.

\section{NumERICAL EXPERIMENTS}

In this section we present some numerical examples that confirm the theoretical findings presented in Sections 2-5. In particular, we check that the convergence rate (5.8) is sharp when the number of sampling points $n$ is chosen as in (5.7).

We consider the elliptic model (1.9) on the bounded domain $D \subset \mathbb{R}^{2}$ with the random diffusion coefficient $a$ defined in (6.1) by means of the geometry displayed in Figure 2. The eight inclusions $D_{1}, \ldots, D_{8}$ are circles with radius equal to 0.13 , and are centered in the points $x=(0.5,0.5 \pm 0.3), x=(0.5 \pm 0.3,0.5)$ and $x=$ $(0.5 \pm 0.3,0.5 \pm 0.3)$. The 0.2 -by- 0.2 inner square $D_{0}$ lies in the center of $D$. The forcing term $f$ is equal to 100 in $D_{0}$ and zero in $D \backslash D_{0}$. The random diffusion coefficient depends on a $d$-dimensional uniform random variable $Y \sim \mathcal{U}\left([-1,1]^{d}\right)$, and is defined as

$$
a(x, y)= \begin{cases}0.395\left(y_{i}+1\right)+0.01, & x \in D_{i}, \quad i=1, \ldots, 8, \forall y \in \Gamma, \\ 1, & x \in D \backslash \cup_{i=1}^{8} D_{i}, \forall y \in \Gamma,\end{cases}
$$

such that each component of the random variable is associated with an inclusion. The range of variation of the coefficient in each inclusion is therefore [0.01,0.8], of course satisfying the uniform ellipticity Assumption 1.11. All inclusions have therefore a similar influence on the solution (isotropic setting). This test case has been used in [3], and allows a direct comparison of our results with those obtained when employing the classical stochastic Galerkin method. The univariate convergence rate $g=1.9$ of this example has been estimated in ([4], Fig. 7-left). 

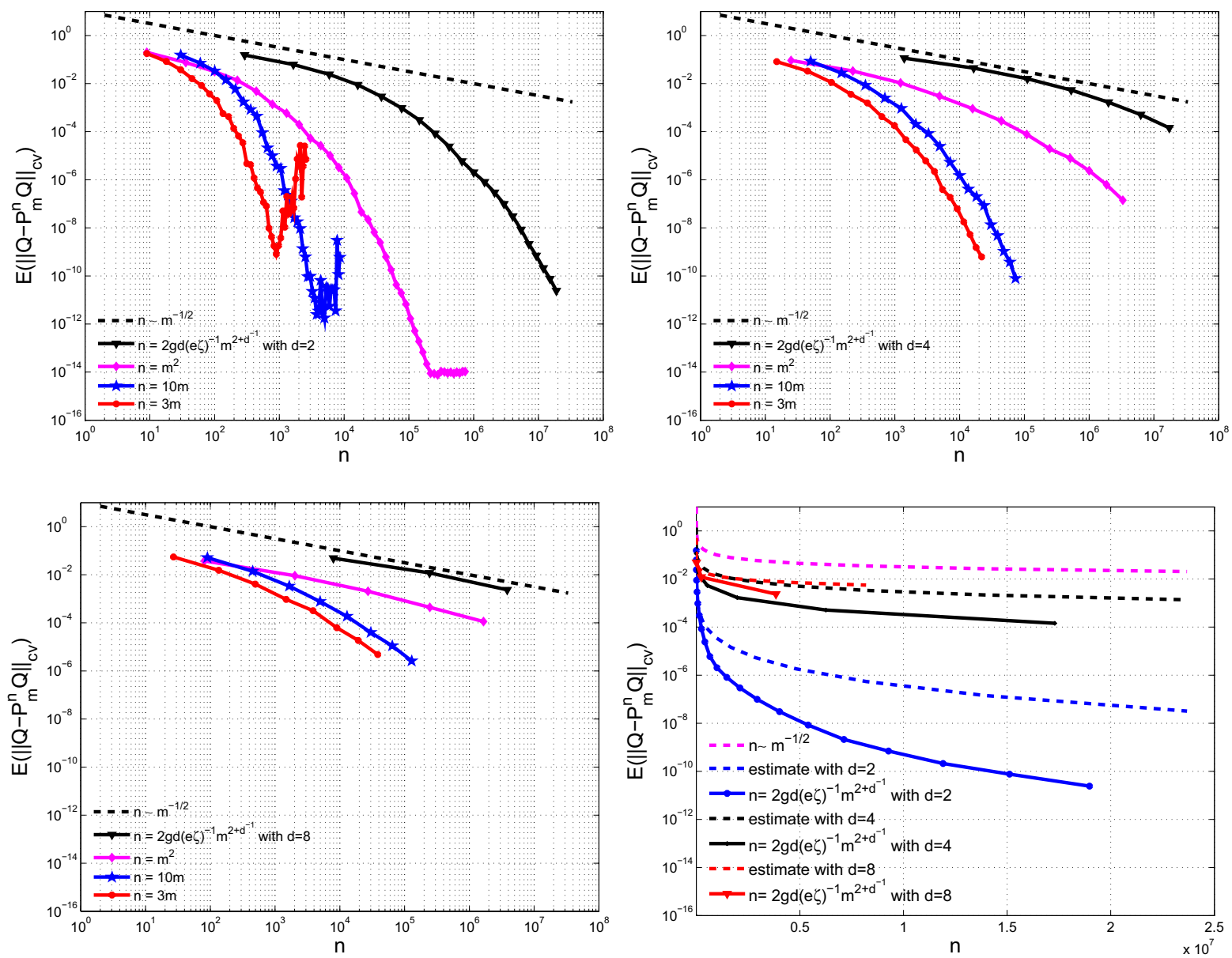

FiguRE 3. Error $\mathbb{E}\left(\left\|Q-\tilde{P}_{m}^{n} Q\right\|_{c v}\right)$, testing different relations between the number of samples $n$ and the dimension of the polynomial space $m$. Top-left: $d=2$. Top-right: $d=4$. Bottom-left: $d=8$. Bottom-right: comparison between the numerical results and the theoretical bound (5.8), with $d=2, d=4$ and $d=8$.

We consider the following quantity of interest related to the solution of the elliptic model (1.9),

$$
Q(u(Y))=\frac{1}{|D|} \int_{D} u(x, y) \mathrm{d} x
$$

and present the results obtained when approximating this function on polynomial spaces of fixed total degree. Similar results hold also with other quantities of interest, see [21]. We consider three cases with $d=2, d=4$, $d=8$ independent random variables. In the case $d=2$, the first random variable describes the diffusion coefficient in the four inclusions at the top, bottom, left, right of the center square $D_{0}$. The second random variable describes the diffusion coefficient in the other four inclusions. In the case $d=4$, each one of the four random variables is associated with two opposite inclusions with respect to the center of the domain. When $d=8$ each one of the random variables is associated with a different inclusion.

Figure 3 shows the convergence plots obtained by the discrete least-squares approximation using a number of samples as in (5.7). The theoretical bound (5.8) is also shown as well as the reference slope $n^{-1 / 2}$ of a standard Monte Carlo method. In the same figures we also show the convergence plots obtained when using a simple linear proportionality $n=3 m$ or $n=10 m$. 
We investigate the behaviour of the $L^{\infty}$ approximation error of the discrete least-squares projection, approximated as

$$
\mathbb{E}\left(\left\|Q(u)-\tilde{P}_{m}^{n} Q(u)\right\|_{\infty}\right) \approx \mathbb{E}\left(\left\|Q(u)-\tilde{P}_{m}^{n} Q(u)\right\|_{c v}\right),
$$

employing the cross-validation procedure described in ([20], Sect. 4) the expectation in the previous formula is estimated by a sample average of the discrete least-squares approximation error using 5 independent random samples of size $n$. The cross-validation error is calculated as

$$
\left\|Q(u)-\tilde{P}_{m}^{n} Q(u)\right\|_{c v}:=\max _{i=1, \ldots, 1000}\left|Q\left(u\left(\tilde{y}_{i}\right)\right)-\tilde{P}_{m}^{n} Q\left(u\left(\tilde{y}_{i}\right)\right)\right|,
$$

where $\left(\tilde{y}_{i}\right)_{1 \leq i \leq 1000}$ is the set of i.i.d. cross-validation points, which is kept fixed among the 5 replicas.

The results presented in Figure 3 show that the theoretical bound (5.8) predicts quite sharply the error $\mathbb{E}\left(\left\|u-\tilde{P}_{m}^{n} u\right\|^{2}\right)$, when the number of sampling points $n$ is chosen according to (5.7). The bound accurately describes the effect of the dimension $d$ as well, in the case of moderately high dimensions.

On the other hand, a faster convergence of the error $\mathbb{E}\left(\left\|u-\tilde{P}_{m}^{n} u\right\|^{2}\right)$ with respect to $n$ is observed, with the linear proportionality $n \sim m$ that yields a lower number of sampling points than (5.7), for a given set 1. The efficiency of the linear proportionality has been pointed out in [21], and its importance is motivated by the impossibility to employ the number of sampling points (5.7) when the dimension $d$ is large. Figure 3 shows that already when $d=8$, the exponential gain of the bound (5.8) with respect to a Monte Carlo rate becomes perceivable only with an astronomical number of samples, making the choice (5.7) less attractive for high-dimensional "isotropic" applications, whereas a linear proportionality, even with $n=3 \mathrm{~m}$ leads to very good results. Observe, however, that a linear proportionality might lead to instability of the discrete least-squares projection as clearly visible in Figure 3 (top-left) in the case $d=2$.

\section{Conclusion}

In this work the approximation technique based on least squares with random evaluations has been analyzed. The condition between the number of sampling points and the dimension of the polynomial space, which is necessary to achieve stability and optimality, has been extended to any lower set of multi-indices identifying the polynomial space, in any dimension of the parameter set, with the uniform and Chebyshev densities and more generally with densities from the beta family. When the measure is uniform, this condition requires the number of sampling points to scale as the square of the dimension of the polynomial space up to logarithmic factors, to achieve optimal convergence rate in expectation or in probability.

As an application of this technique, we have considered a class elliptic PDE models with of "inclusion-type" stochastic coefficients. In this case, exponential convergence rates in expectation can be derived, which require, however, a slightly more demanding relation between the number of sampling points and the dimension of the polynomial space. This estimate clarifies the dependence of the convergence rate on the number of sampling points and on the dimension of the parameter set, and should be compared with the convergence rate of the best $m$-term exact $L^{2}$ projection.

The numerical tests presented show that the proposed estimate is sharp, when the number of sampling points is chosen according to the condition that ensures stability and optimality. In addition, these results show that, in the aforementioned model class, a linear proportionality of the number of sampling points with respect to the dimension seems to be sufficient in high dimension to ensure the stability of the discrete projection, thus leading to faster convergence rates, although we have no rigourous explaination of this fact.

Acknowledgements. F. Nobile and G. Migliorati acknowledge the support of the Center for ADvanced MOdeling Science (CADMOS). R. Tempone is a member of the KAUST SRI Center for Uncertainty Quantification in Computational Science and Engineering. 


\section{REFERENCES}

[1] I. Babuška, F. Nobile and R. Tempone, A stochastic collocation method for elliptic partial differential equations with random input data. SIAM J. Numer. Anal. 45 (2007) 1005-1034.

[2] I. Babuška, R. Tempone and G.E. Zouraris, Galerkin finite element approximations of stochastic elliptic partial differential equations. SIAM J. Numer. Anal. 42 (2004) 800-825.

[3] J. Bäck, F. Nobile, L. Tamellini and R. Tempone, On the optimal polynomial approximation of stochastic PDEs by Galerkin and Collocation methods. Math. Model. Methods Appl. Sci. 22 (2012) 1250023.

[4] J. Beck, F. Nobile, L. Tamellini, R. Tempone, Convergence of quasi-optimal Stochastic Galerkin Methods for a class of PDES with random coefficients. Comput. Math. Appl. 67 (2014) 732-751.

[5] A. Chkifa, A. Cohen, R. DeVore and Ch. Schwab, Sparse adaptive Taylor approximation algorithms for parametric and stochastic elliptic PDEs. Math. Model. Numer. Anal. 47 (2013) 253-280.

[6] A. Chkifa, A. Cohen and Ch. Schwab, High-dimensional adaptive sparse polynomial interpolation and applications to parametric PDEs. Found. Comput. Math. 14 (2014) 601-633.

[7] A. Chkifa, A. Cohen and C. Schwab, Breaking the curse of dimensionality in sparse polynomial approximation of parametric PDEs. To appear in J. Math. Pures Appl. (2015).

[8] A. Cohen, M A. Davenport and D. Leviatan, On the stability and accuracy of least squares approximations. Found. Comput. Math. 13 (2013) 819-834.

[9] A. Cohen, R. DeVore and C. Schwab, Convergence rates of best $N$-term Galerkin approximations for a class of elliptic sPDEs, Found. Comput. Math. 10 (2010) 615-646.

[10] A. Cohen, R. DeVore and C. Schwab, Analytic regularity and polynomial approximation of parametric and stochastic PDE's. Anal. Appl. 9 (2011) 1-37.

[11] D. Coppersmith and T.J. Rivlin, The growth of polynomials bounded at equally spaced points. SIAM J. Math. Anal. 23 (1992) 970-983.

[12] Ph.J. Davis, Interpolation and Approximation. Blaisdell Publishing Company (1963).

[13] C. de Boor and A. Ron, Computational aspects of polynomial interpolation in several variables. Math. Comput. 58 (1992) $705-727$.

[14] C.J. Gittelson, An adaptive stochastic Galerkin method for random elliptic operators. Math. Comput. 82 (2013) 1515-1541.

[15] M. Kleiber and T. D. Hien, The stochastic finite element methods. John Wiley \& Sons, Chichester (1992).

[16] J. Kuntzman, Méthodes numériques - Interpolation, dérivées. Dunod, Paris (1959).

[17] G. Lorentz and R. Lorentz, Solvability problems of bivariate interpolation I. Constructive Approximation 2 (1986) 153-169.

[18] G.Migliorati, Polynomial approximation by means of the random discrete $L^{2}$ projection and application to inverse problems for PDEs with stochastic data. Ph.D. thesis, Dipartimento di Matematica "Francesco Brioschi" Politecnico di Milano, Milano, Italy, and Centre de Mathématiques Appliquées, École Polytechnique, Palaiseau, France (2013).

[19] G. Migliorati, Multivariate Markov-type and Nikolskii-type inequalities for polynomials associated with downward closed multi-index sets. J. Approx. Theory 189 (2015) 137-159.

[20] G. Migliorati, F. Nobile, E. von Schwerin and R. Tempone, Analysis of discrete $L^{2}$ projection on polynomial spaces with random evaluations. Found. Comput. Math. 14 (2014) 419-456.

[21] G. Migliorati, F. Nobile, E. von Schwerin and R. Tempone, Approximation of Quantities of Interest in stochastic PDEs by the random discrete $L^{2}$ projection on polynomial spaces. SIAM J. Sci. Comput. 35 (2013) A1440-A1460.

[22] V. Nistor and Ch. Schwab, High order Galerkin approximations for parametric, second order elliptic partial differential equations, Report 2012-22, Seminar for Applied Mathematics, ETH Zürich. Math. Models Methods Appl. Sci. 23 (2013).

[23] F. Nobile, R. Tempone and C.G. Webster, A sparse grid stochastic collocation method for elliptic partial differential equations with random input data. SIAM J. Num. Anal. 46 (2008) 2309-2345.

[24] F. Nobile, R. Tempone and C.G. Webster, An anisotropic sparse grid stochastic collocation method for elliptic partial differential equations with random input data. SIAM J. Num. Anal. 46 (2008) 2411-2442. 